\title{
The calcium channel Orai1 is required for osteoblast development: studies in a chimeric mouse with variable in vivo Runx-cre deletion of Orai-1
}

Lisa J Robinson, ${ }^{1}$ Jonathan Soboloff, ${ }^{2}$ Irina L Tourkova, ${ }^{3}$ Quitterie C Larrouture, ${ }^{3}$ Dionysios J Papachristou, ${ }^{3,4}$

5 Scott Gross, ${ }^{2}$ Robert Hooper, ${ }^{2 \# a}$ Elsie Samakai, ${ }^{2 \# b}$ Paul F Worley, ${ }^{5}$ Jan Tuckermann, ${ }^{6}$ Michelle R Witt, ${ }^{1}$ Harry C Blair $3^{*}$

1Departments of Pathology, Anatomy and Laboratory Medicine, and of Microbiology, Immunology \& Cell Biology, West Virginia University School of Medicine, Morgantown, WV 26506 USA

10 '2Fels Cancer Institute for Personalized Medicine, Department of Cancer and Cellular Biology, Lewis Katz School of Medicine at Temple University, Philadelphia, PA 19140 USA

${ }^{3}$ Departments of Pathology and of Cell Biology, the Pittsburgh VA Medical Center and the University of Pittsburgh, Pittsburgh PA 15261 USA

${ }^{4}$ Laboratory of Bone and Soft Tissue Studies, Department of Anatomy-Histology-Embryology, University Patras

15 Medical School, Patras, Greece

${ }^{5}$ Solomon H. Snyder Department of Neuroscience, Johns Hopkins University School of Medicine, Baltimore, MD 21205 USA

${ }^{6}$ Institute of Comparative Molecular Endocrinology, Helmholtzstraße 8/1, 89081 Ulm, Germany

\#a

20 Current Address: Rockland Immunochemicals Inc., Jones Blvd, Limerick, PA 19468 USA

\#b Current Address: Exploratory Biology group, Janssen Pharmaceutical, Spring House, PA 19477 USA

Short Title: The calcium channel Orai 1 in osteoblast development

25 * Corresponding Author:

E-mail hcblair@pitt.edu (HCB) 


\section{Abstract}

The calcium-selective ion channel Orai1 has a complex role in bone homeostasis, with defects in both bone production and resorption detected in Orai1 germline knock-out mice. To determine whether Orai1 has a 30 direct, cell-intrinsic role in osteoblast differentiation and function, we bred Orai1 flox/flox (Orai1/f/f) mice with Runx2-cre mice to eliminate its expression in osteoprogenitor cells. Interestingly, Orai1 was expressed in a mosaic pattern in Orai1/f/f-Runx2-cre bone. Specifically, antibody labeling for Orai1 in vertebral sections was uniform in wild type animals, but patchy regions in Orai $1^{\mathrm{f} / \mathrm{f}}$-Runx2-cre bone revealed Orai1 loss while in other areas expression persisted. Nevertheless, by micro-CT, bones from Orai $1^{\mathrm{ff}}$-Runx2-cre mice showed reduced

35 bone mass overall, with impaired bone formation identified by dynamic histomorphometry. Cortical surfaces of Orai 1/f/-Runx2-cre vertebrae however exhibited patchy defects. In cell culture, Orai1-negative osteoblasts showed profound reductions in store-operated $\mathrm{Ca}^{2+}$ entry, exhibited decreased alkaline phosphatase activity, and had markedly impaired substrate mineralization. We conclude that defective bone formation observed in the absence of Orai1 reflects an intrinsic role for Orai1 in differentiating osteoblasts. 
Introduction

Orai1 is a calcium-selective ion channel found in the plasma membrane, activated by STIM1, a calcium sensor located on the endoplasmic reticulum [1, 2, 3]. Although expressed in all cells, the extent to which different cell types require Orai1 is variable. For example, T cell activation requires Orai1; its loss causes severe combined immunodeficiency [4,5]. Loss of Orai1 also causes a wide range of changes in cardiovascular function [6], neuronal function [7, 8], tooth formation [9, 10] and others [11]. We [12, 13, 14, 15, $16]$ and others [17, 18], demonstrated that Orai 1 contributes to bone differentiation and maintenance in conventional knockout mice and in vitro cell lines.

Investigation of mice with global deletion of Orai1 revealed loss of multinucleated osteoclasts, that, intriguingly, did not lead to osteopetrosis: Instead, micro-computed tomography showed reduced cortical ossification and thinned trabeculae in Orai $1^{--}$animals compared with controls [12]. We attributed the unexpected finding of reduced osteoclast formation without increased bone density to a probable defect in bone formation by osteoblasts. However, considering the near-lethal phenotype of Orai $1^{-/-}$mice [11] and the interdependence of osteoclast and osteoblast differentiation, these investigations were insufficient to draw definitive conclusions regarding the role of Orai1 within bone-forming osteoblasts specifically.

Here, we assessed the role of Orai1 on osteoblast differentiation in vivo using a conditional knockout mouse. Interestingly, we found that Orai1 deletion in vivo was highly regional, creating a mosaic effect within bone. Irrespective, loss of Orai1 eliminated store-operated $\mathrm{Ca}^{2+}$ entry in osteoblasts and precursors resulted in a severe defect in osteoblast differentiation and function. Altogether, this investigation shows that Orai1 is required for normal osteoblast function. 


\section{Materials and Methods}

Animals and Genotyping. Orai $1^{\text {fl/fl }}$ mice were generated by flanking Orai1 exons $2 / 3$ with loxP as previously described [16, 19]. Mice were backcrossed to C57BI/6 for a minimum of 10 generations and then crossed with Runx2-cre mice ( $\operatorname{Tg}($ Runx2-icre)1Jtuc) [20] for conditional deletion in the osteoblast lineage. Orai ${ }^{\text {fl/fl }}$ mice were identified by PCR of genomic DNA from tail snips using the following primers: flox-F: ACC CAT GTG GTG GAA AGA AA and flox-R: TGC AGG CAC TAA AGA CGA TG; these generate a 746 bp product in wildtype mice and a 505 bp product in Orai $1^{\text {fl/fl }}$ mice [16]. To confirm excision of Orai1 from bone cells, PCR of genomic DNA was performed using the flox-F primer paired with excision-R: CAG AAA GAA CTA CAC AGA GAA ATC, as described [16]; excision results in a 520 bp product, while none is produced when the gene is intact. Mice were sacrificed at 16 weeks unless otherwise noted. For dynamic histomorphometry, animals were 70 injected with xylenol orange ( $80 \mu \mathrm{g} / \mathrm{g}$ mouse weight) five days prior to sacrifice, then with calcein (20 $\mu \mathrm{g} / \mathrm{g}$ mouse weight) 2 days later. Mouse long bones were dissected, cleaned of soft tissue, and processed for isolation of mesenchymal stem cells and osteoblasts. The vertebral column was removed for histologic and microCT analysis. Work was approved by the Temple University IACUC.

Cell isolation and differentiation. Unless stated, media and chemicals were from Thermo-Fisher. Mesenchymal stem cells (MSC) were isolated from long bones as described [21]. Briefly, marrow was flushed with Minimum Essential Medium-alpha (alphaMEM) containing 10\% fetal bovine serum (Sigma-Aldrich, St. Louis, MO), penicillin and streptomycin. After plating cells 16 hours to allow adhesion of stromal cells, nonadherent cells were removed, and the adherent cells grown in selective medium (Mesencult, StemCell Technologies, Cambridge, MA), at $2 \times 10^{6} \mathrm{cells} / \mathrm{cm}^{2}$, for proliferation of MSC. Osteoblasts were isolated from

80 long bones as described [22]: briefly, after removal of marrow, the long bones, cut into 1-2 mm fragments, were incubated in $2 \mathrm{mg} / \mathrm{ml}$ collagenase type II (260 U/mg, Worthington, Lakewood, NJ) in Dulbecco's Modified Eagle's Medium (DMEM) for 2 hours at $37^{\circ} \mathrm{C}$. The bone fragments were then rinsed and transferred to culture dishes with Osteoblast Growth Medium (DMEM with $1 \mathrm{~g} / \mathrm{l}$ glucose, 10\% heat inactivated fetal bovine serum, 30 $\mu \mathrm{g} / \mathrm{ml}$ ascorbate, penicillin, streptomycin, and amphotericin B. For differentiation to osteoblasts with mineralized matrix formation, MSC or osteoblasts at confluence were transferred to Osteoblast Mineralization Medium (Osteoblast Growth medium with $30 \mu \mathrm{g} / \mathrm{ml}$ ascorbate and $10 \mathrm{mM}$ 2-glycerol phosphate). During culture, media were replaced every 2-3 days.

Histomorphometry and Histology. Static histomorphometry was performed as described [16]. Briefly, lumbar vertebrae were fixed overnight in 3.7\% formalin, then transferred to $70 \%$ ethanol for micro-CT analysis 90 using Bruker Skyscan 1272 with a bone density cutoff of $150 \mathrm{mg} / \mathrm{cm}^{2}$, at $5 \mu \mathrm{m}$ resolution. Scans were analyzed using Bruker CTan software for trabecular parameters; three-dimensional images were produced with Bruker CTvox software. For dynamic histomorphometry, vertebral sections were taken from animals labeled with xylenol orange and calcein two days apart. Lumbar vertebrae 1-3 were used for dynamic histomorphometry and histologic studies, and vertebrae 4-6 were for micro-computed tomography. Bone samples for fluorescent microscopy were cut without decalcification. For hematoxylin and eosin staining, bone was fixed, dehydrated, 
paraffin embedded, and cut as $10 \mu \mathrm{m}$ thick sections using a rotary microtome [23]. Cortical thickness was measured orthogonal to the vertical axis in microns at $300 \mu \mathrm{m}$ intervals; dynamic histomorphometry was as described using calcein and xylenol orange labels [23]. Labeling was analyzed by observers blinded to genotype.

Alkaline phosphatase, mineralization, and adipocyte assays. Mineral was labeled with silver nitrate (von Kossa stain): cultures were rinsed with water and fixed with 3.7\% formalin for 2 minutes, then incubated with $2 \% \mathrm{AgNO}_{3}$ under UV light for 10 minutes, and rinsed again with water. Alkaline phosphatase activity was determined in cell cultures using $0.01 \%$ naphthol phosphate substrate in citrate-buffered saline at pH 8 plus $0.25 \mathrm{mg} / \mathrm{ml}$ of fast blue to precipitate an insoluble blue adduct [24]. Parallel cultures were assessed for adipocyte formation by incubating fixed cells in $0.3 \%$ Oil red $\mathrm{O}$ in $60 \%$ isopropanol for 1 hour and rinsed with water.

Measurement of Store Operated Calcium Entry. Osteoblasts on glass coverslips were loaded with 2 $\mu \mathrm{M}$ Fura-2 AM-ester (Invitrogen) for 30 minutes at $25^{\circ} \mathrm{C}$ in $107 \mathrm{mM} \mathrm{NaCl}, 7.2 \mathrm{mM} \mathrm{KCl}, 1.2 \mathrm{mM} \mathrm{MgCl}, 11.5$ $\mathrm{mM}$ glucose, $20 \mathrm{mM} \mathrm{HEPES,} 1 \mathrm{mM} \mathrm{CaCl}_{2}$, at $\mathrm{pH}$ 7.2. Washed cells were allowed to de-esterify dye for 30 minutes at $25^{\circ} \mathrm{C}$ as described [16]. $\mathrm{Ca}^{2+}$ measurements used a Leica DMI 6000B fluorescence microscope controlled by Slidebook Software (Intelligent Imaging; Denver, CO). Intracellular Ca ${ }^{2+}$ is shown as $340 / 380 \mathrm{~nm}$ ratios from single cells. Data used 15-20 cells per mouse and three or more experiments.

Orai1 labeling. Rabbit polyclonal-anti Orai1 was used for tissue labeling [25]; rabbit anti-Orai1 (extracellular) antibody was from Alomone Labs (ACC-062, Jerusalem, Israel). Triplicate sections from three 115 wild type and three knockout animals (to allow no-antibody controls) of wild type (WT) and knockout bone (KO) were de-paraffinized by $67^{\circ} \mathrm{C}$ heating for two days followed by a xylene rinse 12 hours. Labeling was done twice with different sections. In each case, sections were hydrated with $70 \%$ ethanol followed by PBS with $1 \%$ bovine serum albumin (BSA) with $2 \mathrm{mM}$ EDTA (blocking solution) overnight at room temperature, followed by antibody at 1:100 in PBS with BSA and EDTA (or no antibody controls) overnight at room temperature, and then secondary labeling with anti-rabbit Alexafluor 488 (green) for four hours at room temperature, followed by rinsing and fixation with 3.7\% formalin in PBS. When indicated, post staining with phalloidin-rhodamine (1:100) was used to label actin. For Western blots, cell lysates were made using $0.3 \%$ SDS, $50 \mathrm{mM}$ tris, $\mathrm{pH} 7$, with proteinase, and phosphatase inhibitors. Proteins were separated on 4-12\% gradient gel and transferred to polyvinylidene difluoride (PVDF) membranes. The primary antibody was the Alomone antibody used for

125 fluorescence (1:200); secondary antibody was horse radish peroxidase conjugated anti-rabbit (1:40,000, Jackson ImmunoResearch, West Grove, PA). Proteins on blots were detected by enhanced chemiluminescence detection (ECL plus, Amersham, Piscataway, NJ, USA). Mouse beta-actin antibody $(1: 1,000$, Sigma) with secondary horse radish peroxidase conjugated anti-mouse $(1: 40,000)$ were controls.

Statistics. Results are mean \pm SD, for three or more measurements or as stated. Comparisons of differences used Student's unpaired t-test. Significance indicates $p<0.05$. 


\section{Results}

Phenotype of wild type and Orai1 ${ }^{\mathrm{f} / f_{-}}$Runx2-cre animals. Orai ${ }^{\mathrm{flf}}$-Runx2-cre conditional knockout mice revealed no gross differences in size, health or behavior compared to controls. Histo-morphometric studies of vertebrae were performed for detailed assessment of the bone. Wild type vertebrae had typical morphology (examples are shown, Fig 1A), while Orai $1^{\mathrm{f} / \mathrm{f}}$-Runx2-cre vertebrae showed a patchy reduction in cortical bone (examples are shown, Fig 1B). To better understand the basis for this distinct pock-marked appearance in Orai $1^{\mathrm{ff}}$-Runx2-cre animals, cortical bone was examined in cross section. The appearance of the wild type cortex was unremarkable, but cortical bone in Orai1//f-Runx2-cre animals showed irregular thinning (Fig 2A-B). This lack of regularity is unusual but was observed in three of three Orai ${ }^{1 / f}-$ Runx2-cre and none of three wild type animals analyzed. Analysis of measurements of cortical thickness, in microns at $300 \mu \mathrm{m}$ intervals, showed that cortical bone was significantly thinned in the Orai $1^{\mathrm{flf}}$-Runx2-cre animals (Fig 2C). We hypothesized that areas of reduced bone reflected regions in which Orai1 had been deleted by cre-recombination thus impairing bone formation, but that cre-mediated excision was incomplete with areas of normal thickness reflecting preserved bone formation by osteons with Orai1 positive cells. This was further investigated by antibody labeling for Orai1 (see below).

Fig 1. Surface of wild type and Oraif fl/fl_Runx2cre fourth lumbar vertebrae. Bruker CTvox software-generated threedimensional images of vertebrae reconstructed from microCT scans at $5 \mu \mathrm{m}$ resolution. All animals were homozygous for floxed Orai1; the conditional knockouts (lower panels) are Runx2-cre positive.

150 A. Representative vertebrae from control animals. Apart from sites of blood vessel entry, the surface of the bone is smooth, typical for mice at four months of age.

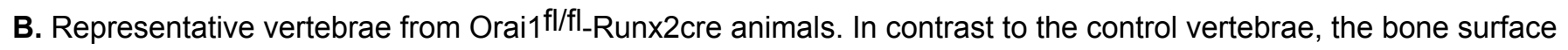
appears irregular with patchy darker areas representing regions of reduced bone.

Fig 2. Cross sections of vertebral cortex from wild-type (A) and Orai $1^{f / f}$-Runx2-cre conditional $K O(B)$ animals. Images of H\&E stained histologic sections from upper lumbar vertebrae (top panels) are $1 \mathrm{~mm}$ wide; images from microCT scans of lower lumbar vertebrae (middle and lower panels) are $1.4 \mathrm{~mm}$ wide, with trabecular bone deleted.

A. Wild type cortex with typical smooth bone showed relatively uniform thickness.

B. The Orai 1 fl/fl-Runx2cre (abbreviated Orai1 $\mathrm{cKO}$ ) animals had irregularly thinned regions (arrow) but no other distinguishing features. This is in keeping with the appearance of the bone surface in the three-dimensional reconstructions (see Fig 1).

C. Cortical bone thickness, though variable, is reduced on average in Orai1 $1^{f l / f l}$-Runx2cre (Orai1 cKO) animals $(p=0.009$, $\mathrm{N}=8$ ). 
that overall bone mass, measured as bone volume/tissue volume (BV/TV), was reduced in Orai $1^{\mathrm{ff}}$-Runx2-cre animals compared to controls (Fig 3A; $p=0.01$ ). Mean trabecular thickness was also decreased (Fig 3B; $p<0.01$ ) in Orai $1^{\mathrm{f} / \mathrm{f}}$-Runx2-cre bone, as was trabecular number (Fig 3C, $\mathrm{p}<0.02$ ) in the Orai1 conditional knockouts. Antemortem fluorescent labeling of newly formed bone by injected calcein and xylenol orange was used for dynamic histomorphometry studies. Consistent with the patchy thinner and thicker areas of bone noted above (Figs 1 and 2) suspected to reflect regions of Orai1 negative and positive osteoblasts, we found a patchy distribution of new bone formation with calcein/xylenol orange labeling (Fig 3D) in the Orai1 conditional knock-out animals. This irregular labeling does not fit traditional histomorphometric parameters and was found in none of the controls, but was consistent across the Orai $1^{\mathrm{flf}}$-Runx2-cre samples examined. In regions where labeling was intact, dynamic histomorphometric measurements at time of sacrifice showed no difference in bone apposition rate (interlabel distance, not shown) or fraction of labeled bone surface (Fig 3E). Finally, despite the irregularity of the phenotype, the calcein labeling did demonstrate reduction in bone deposition overall in Orai1 ${ }^{\mathrm{f} / \mathrm{f}}$-Runx2-cre mice (Fig 3F, $p=0.03$ ). This indicates that bone forming regions in Orai ${ }^{\mathrm{f} / \mathrm{f}}$-Runx2cre mice were smaller than in control animals. Given the patchy nature of these effects, we suspect that the contribution of Orai1 to bone formation in affected regions may be variable. To determine if indeed variable Orai 1 deletion is the cause of this variable phenotype, we assessed Orai 1 deletion further.

Fig 3. Key features of WT and conditional KO animals on static and dynamic histomorphometry.

185 A. Bone volume/total volume (BV/TV) of vertebrae. Vertebral bone is significantly reduced $(N=7, p=0.01)$ in the Orai $1^{f l / f l}$. Runx2cre (Orai1 cKO) mice compared to controls. The greater variability in the conditional-knockout bone is thought to reflect variable cre excision; this variability is also apparent in the cortical section thickness (Fig 2; see text).

B. Trabecular thickness is significantly decreased in the Orai1 conditional knockout mice $(\mathrm{N}=7, \mathrm{p}<0.01)$.

C. Trabecular number is also significantly decreased in the conditional knockouts $(N=7, p<0.02)$.

D. Example of xylenol orange (red) and calcein (green) fluorescent labels in wild type and conditional KO bones. The interlabel distance was not statistically different (not illustrated). Fields shown are $100 \mu \mathrm{m}$ wide.

E. The proportion of calcein labeled bone surface in wild type and Orai $1 \mathrm{fl} / \mathrm{fl}-\mathrm{Runx} 2 \mathrm{cre}$ bone (abbreviated Orai1 cKO) was not statistically different $(\mathrm{N}=4)$.

F. The bone formation rate at 4 months of age was significantly reduced in the Orai $1^{\mathrm{fl} / \mathrm{fl}}$-Runx2cre mice (Orai1 $\left.\mathrm{cKO}\right)$ compared to controls $(\mathrm{N}=4, \mathrm{p}=0.03)$.

Variable deletion of osteoblast flox/flox Orai1 by the Runx2-cre promoter. Incomplete deletion by Cre has been demonstrated in many different mouse models (see Discussion), although we found the apparent mosaic phenotype of this particular mouse striking. To demonstrate variable deletion of Orai 1 within bone cells, we performed antibody labeling using an Orai1 antibody in wild type and Orai1/f/Runx2-cre bone (Fig 4). Controls for specificity of labeling are shown (Fig 4A). While all WT bone cells expressed Orai1 (Fig 4A), there were patches of Orai1-negative (arrows) as well as Orai1-positive osteocytes in Orai1 ${ }^{\mathrm{f} / \mathrm{f}-\mathrm{Runx} 2 \text {-cre }}$ 
mice (Fig 4B and 4C, lower panels). Western blot showed significant but incomplete Orai1 deletion in cultures of Orai1 $1^{\mathrm{f} / \mathrm{f}}$-Runx2-cre osteoblasts (Fig 4D). This was consistent variable deletion of Orai1 in osteoblasts from 205 Orai $1^{\mathrm{f} / f}$-Runx2-cre mice. To determine the functional implications of Orai1 deletion in osteoblasts, in vitro cell cultures, negative for Orai1 by PCR, in mineralization medium were used, as described below.

Fig 4. Variable efficacy of Runx2-cre in deleting flox/flox Orai1 in osteoblasts. It is often assumed that promoter-cre constructs uniformly delete the target in all cells of an organ. Recent findings suggest that for reasons that are not clear

210 this is sometimes not the case (see Text), we tested this by antibody labeling of Orai1 in wild type and Orai 1 fl/fl-Runx2cre bone.

A. Rabbit antibody detects Orai1 in osteoblasts (upper left panel). Absence of antibody eliminates labeling (lower left panel). All sections are from one wild type animal. Left and right panels are of the same section. Osteoblasts are shown independently with phalloidin rhodamine (right panels). Fields are $200 \mu \mathrm{m}$ across.

215 B. In the wild type animal, osteoblasts are shown with the antibody at high power (fields 200 microns wide) and in phase of the same field (right). In the lower panels, the same field of a conditional KO animal. Some of the conditional KO cells (Orai1 $1^{f l / f l}-$ Runx2cre) do not label (arrows, phase and antibody label, lower panels).

C. Lower power fields, $350 \mu \mathrm{m}$, of Orai1 labeled osteoblasts (surrounding tissue fluorescence is an artifact) in wild type bone (top) and conditional KO bone (bottom). Osteoblasts in the wild type label strongly, including surface cells of the bone (arrows, top). Some, but not all, of the osteoblasts in the conditional KO label (arrows, bottom).

D. Western blot for Orai1 in cells expressing or not expressing the protein (See Fig 5). Thirty-five $\mu$ g loads of cell protein from the isolates indicated were run on denaturing SDS-PAGE and blotted. This blot using the very specific Alomone antibody shows a trace of Orai1 even in the osteoblast KO preparation (left lane) and reduced, but not absent, Orai1 in the MSC KO (upper panel). The beta actin re-blot (lower panel) confirms similar protein loads.

Effect of Orai1 deletion on store-operated calcium entry. MSCs were collected from Orai $1 \mathrm{fl} / \mathrm{fl}$ and Orai ${ }^{1 / f}$-Runx2-cre mice before differentiating into osteoblasts in vitro and measuring store-operated calcium entry as described [16]. Cells were treated with thapsigargin in the absence of extracellular calcium to deplete the endoplasmic reticulum of calcium and initiate the store-operated calcium response. While a typical storeoperated calcium response was observed in Orai1-expressing osteoblasts, loss of Orai1 completely eliminated store-operated calcium entry (Fig 5). These observations show that Orai1 is required for store-operated calcium entry in osteoblasts.

Fig 5. Calcium activated calcium release in cells from wild type and Runx2cre-Oraifl/fl mice. Intracellular calcium was measured by Fura2 (Wang, 2009) (top panel). Cells were treated with thapsigargin in the absence of extracellular calcium. Only the wild type cells show calcium entry upon extracellular calcium repletion. Examples of Fura2 signals from control and conditional KO cells are shown in the middle and lower panels respectively, with false color reflecting calcium concentration in cells (scale is shown in upper right corner of each panel). A scale bar for size for the photomicrographs is 
shown below the bottom panel.

Orai1 $^{f / f}$-Runx2-cre cells showed severe defects in osteoblast differentiation. We analyzed osteoblast differentiation using wild type cells and cells from conditional knockouts in which absence of Orai1 was confirmed by PCR (not illustrated), grown in mineralization medium with 2-glycerol phosphate and ascorbate. Mineral deposition was detected using silver nitrate (von Kossa); mineralization was prominent in cultures of wild type cells, but markedly reduced for Orai $1^{\mathrm{f} / \mathrm{f}}$-Runx2-cre osteoblasts (Fig 6A). Additionally, alkaline phosphatase positivity in the conditional KO was significantly decreased relative to wild type (Fig 6B). Reduced osteoblastic differentiation correlates with increased adipocytes in mice fed a Western diet [26]. However, no Orai1-dependent differences in adipogenic differentiation were identified by Oil red $\mathrm{O}$ staining in these culture conditions (Fig 6C), suggesting that Orai1 expression was did not favor osteoblast-adipocyte conversion. For definitive evaluation of the role of Orai1 further studies using adipogenic culture conditions would be needed.

\section{Fig 6. Elimination of Orai1 results in profoundly reduces $O B$ differentiation and mineralization from $O B$}

precursors. Osteoblasts isolated as described in the methods section from control or Runx2-cre floxed (Orai1 ${ }^{\text {f/f }}$-Runx2cre) conditional knock-out animals, were incubated 24 days in osteoblast mineralization medium and analyzed by histologic staining. Each well illustrated is $2 \mathrm{~cm}$ across.

A. Von Kossa staining for mineral. Wild type cells made mineral nodules, but there were only rare and small nodules in Orai1 knockout cell cultures. Representative culture wells for control (WT) and conditional knockout (cKO) cells are shown on the left. Staining was quantified for four samples of each genotype. Mineralized matrix production appeared significantly reduced in cultures of Orai1-deficient osteoblasts $(p<0.0001, N=4)$.

B. Alkaline phosphatase activity. Representative cultures are shown on the left. Results quantified from four samples of each genotype are shown on the right. Wild type cells produced much more alkaline phosphatase, with small nodules of high activity as in the silver stain for mineral $(A)$, there was uniformly less alkaline phosphatase in Orai1 null cells $(p=0.0002, N=4)$.

265 C. Oil red O staining for adipocytes. Representative cultures are shown on the left. Staining was quantified for four samples of each genotype with results shown on the right. There was no evidence of increased adipogenic differentiation in Orai1 null cells, with minimal labeling in cultures of either genotype. 


\section{Discussion}

We show that Orai1 serves an essential cell-intrinsic function in osteoblast differentiation. Interpretation is complicated by incomplete deletion of Orai1, resulting in animals exhibiting an intriguing mosaic phenotype. Interestingly, complete deletion of glucocorticoid genes early in osteoblast differentiation has been reported in Runx2-cre mice [20]. While the reasons for this difference are not clear; epigenetic differences in the status of the Orai1 gene amongst different osteoblasts represent one possible explanation; silencing of genes due to histone methylation have been shown to interfere with cre recombinases in prior study [27]. While this possibility has not been established for the Runx2-cre floxed Orai1 combination, it would be consistent with the findings reported here and a subject for future study. In keeping, other studies have shown incomplete cremediated excision [28, 29].

Global analysis of bone density showed significant but subtle differences. We feel that this likely reflects the incomplete deletion of Orai1 in vivo. However, in vitro bone differentiation from Orai $1^{-/-}$cells caused profound reductions in osteoblast differentiation. This reflects that cells used were either wild type or confirmed Orai ${ }^{-/-}$, while in vivo a patchwork of normal and Orai $1^{-/-}$cells occurs.

Further, while Runx2 is crucial for the commitment of mesenchymal stem cells to the osteoblast lineage, Runx2 expression is down-regulated at later stages [30]. While there is convincing evidence that Orai1 is involved in osteoblast differentiation and function in the current study and in [31], the uncertain status of Orai 1 in osteoblast precursors combined with the temporary nature of Runx2 expression in vivo may contribute to the mosaic phenotype in this model.

The present work using non-transformed cells indicates unequivocally the essential role of Orai1 in bone differentiation. When Orai1 is eliminated in pre-osteoblastic cells, limited osteoblast differentiation occurs, with remarkable variability of osteoblast-related differentiation. The ability of osteoblasts to form mineralizing nodules is completely abrogated in Orai1-null osteoblasts. This is consistent with the necessity of storeoperated calcium entry for bone differentiation. This further suggests that the formation of advanced osteoblast-related structures [32] is impossible without store-operated calcium entry. There are a many studies indicating importance for calcium signals in bone formation, e.g., [33, 34, 35], but previously not showing specificity for Orai1 or calcium-activated calcium release in normal osteoblast differentiation. Our work is cell specific, unlike general Orai1 knockout studies [12, 31], in which bone degrading, bone forming, and potentially other cell types were affected. In truly Orai1 negative osteoblasts, bone differentiation is severely compromised.

\section{Acknowledgements}

We thank Cayla R Sudano and Kelechi M Onwuka for assistance. 


\section{References}

1. Soboloff J, Rothberg BS, Madesh M, Gill DL. STIM proteins: dynamic calcium signal transducers. Nat Rev

Mol Cell Biol. 2012;13(9):549-565. doi:10.1038/nrm3414

2. Hogan PG, Lewis RS, Rao A. Molecular basis of calcium signaling in lymphocytes: STIM and ORAI. Annu Rev Immunol. 2010;28:491-533. doi:10.1146/annurev.immunol.021908.132550

3. Prakriya M, Lewis RS. Store-Operated Calcium Channels. Physiol Rev. 2015;95(4):1383-1436. doi:10.1152/physrev.00020.2014

310 4. Feske S, Gwack Y, Prakriya M, Srikanth S, Puppel SH, Tanasa B, et al. A mutation in Orai1 causes immune deficiency by abrogating CRAC channel function. Nature. 2006;441(7090):179-185. doi:10.1038/nature04702

5. Oh-Hora M, Yamashita M, Hogan PG, Sharma S, Lamperti E, Chung W, et al. Dual functions for the endoplasmic reticulum calcium sensors STIM1 and STIM2 in T cell activation and tolerance. Nat Immunol. 2008;9(4):432-443. doi:10.1038/ni1574

6. Tanwar J, Trebak M, Motiani RK. Cardiovascular and Hemostatic Disorders: Role of STIM and Orai Proteins in Vascular Disorders. Adv Exp Med Biol. 2017;993:425-452. doi:10.1007/978-3-319-57732-6_22

7. Hartmann J, Karl RM, Alexander RP, Adelsberger H, Brill MS, Rühlmann C, et al. STIM1 controls neuronal $\mathrm{Ca}^{2+}$ signaling, mGluR1-dependent synaptic transmission, and cerebellar motor behavior. Neuron. 2014;82(3):635-644. doi:10.1016/j.neuron.2014.03.027

8. Lalonde J, Saia G, Gill G. Store-operated calcium entry promotes the degradation of the transcription factor Sp4 in resting neurons. Sci Signal. 2014;7(328):ra51. doi:10.1126/scisignal.2005242

9. Nurbaeva MK, Eckstein M, Concepcion AR, Smith CE, Srikanth S, et al. Dental enamel cells express functional SOCE channels. Sci Rep. 2015;5:15803. doi:10.1038/srep15803

10. Eckstein M, Lacruz RS. CRAC channels in dental enamel cells. Cell Calcium. 2018;75:14-20. doi:10.1016/j.ceca.2018.07.012

11. Gwack Y, Srikanth S, Oh-Hora M, Hogan PG, Lamperti ED, Yamashita M, et al. Hair loss and defective Tand B-cell function in mice lacking ORAI1. Mol Cell Biol. 2008;28(17):5209-5222. doi:10.1128/MCB.00360-08

12. Robinson LJ, Mancarella S, Songsawad D, Tourkova IL, Barnett JB, Gill DL, et al. Gene disruption of the calcium channel Orai1 results in inhibition of osteoclast and osteoblast differentiation and impairs skeletal development. Lab Invest. 2012;92(7):1071-1083. doi:10.1038/labinvest.2012.72

13. Zhou Y, Lewis TL, Robinson LJ, Brundage KM, Schafer R, Martin KH, et al. The role of calcium release activated calcium channels in osteoclast differentiation. J Cell Physiol. 2011;226(4):1082-1089. 
doi:10.1002/jcp.22423 doi:10.1002/jcp.22423

14. Blair HC, Soboloff J, Robinson LJ, Tourkova IL, Larrouture QC, Witt MR, et al. Suppression of arthritis-

induced bone erosion by a CRAC channel antagonist. RMD Open. 2016;2(1):e000093. doi:10.1136/rmdopen2015-000093

15. Robinson LJ, Blair HC, Barnett JB, Soboloff J. The roles of Orai and Stim in bone health and disease. Cell Calcium. 2019;81:51-58. doi:10.1016/j.ceca.2019.06.001

16. Robinson LJ, Soboloff J, Tourkova IL, Larrouture QC, Witt MR, Gross S, et al. The function of the calcium channel Orai1 in osteoclast development. FASEB J. 2021;35(6):e21653. doi:10.1096/fj.202001921RR

17. Kim MS, Yang YM, Son A, Tian YS, Lee SI, Kang SW, et al. RANKL-mediated reactive oxygen species pathway that induces long lasting $\mathrm{Ca} 2+$ oscillations essential for osteoclastogenesis. J Biol Chem. 2010;285(10):6913-6921. doi:10.1074/jbc.M109.051557

18. Hwang SY, Putney JW. Orai1-mediated calcium entry plays a critical role in osteoclast differentiation and 345 function by regulating activation of the transcription factor NFATc1. FASEB J. 2012;26(4):1484-1492. doi:10.1096/fj.11-194399

19. Ahuja M, Schwartz DM, Tandon M, Son A, Zeng M, Swaim W, et al. Orai1-Mediated Antimicrobial Secretion from Pancreatic Acini Shapes the Gut Microbiome and Regulates Gut Innate Immunity. Cell Metab. 2017;25(3):635-646. doi:10.1016/j.cmet.2017.02.007

20. Rauch A, Seitz S, Baschant U, Schilling AF, Illing A, Stride B, et al. Glucocorticoids suppress bone formation by attenuating osteoblast differentiation via the monomeric glucocorticoid receptor. Cell Metab. 2010;11(6):517-531. doi:10.1016/j.cmet.2010.05.005

21. Dobrowolski SF, Tourkova IL, Robinson LJ, Secunda C, Spridik K, Blair HC. A bone mineralization defect in the Pahenu2 model of classical phenylketonuria involves compromised mesenchymal stem cell differentiation. Mol Genet Metab. 2018;125(3):193-199. doi:10.1016/j.ymgme.2018.08.010

22. Bakker AD, Klein-Nulend J. Osteoblast isolation from murine calvaria and long bones. Methods Mol Biol. 2012;816:19-29. doi:10.1007/978-1-61779-415-5_2

23. Blair HC, Kalyvioti E, Papachristou NI, Tourkova IL, Syggelos SA, Deligianni D, et al. Apolipoprotein A-1 regulates osteoblast and lipoblast precursor cells in mice. Lab Invest. 2016;96(7):763-772. doi:10.1038/labinvest.2016.51

24. Tourkova IL, Liu L, Sutjarit N, Larrouture QC, Luo J, Robinson LJ, Blair HC. Adrenocorticotropic hormone and 1,25-dihydroxyvitamin D3 enhance human osteogenesis in vitro by synergistically accelerating the expression of bone-specific genes. Lab Invest. 2017;97(9):1072-1083. doi:10.1038/labinvest.2017.62 
25. Ritchie MF, Yue C, Zhou Y, Houghton PJ, Soboloff J. Wilms tumor suppressor 1 (WT1) and early growth response 1 (EGR1) are regulators of STIM1 expression. J Biol Chem. 2010;285(14):10591-10596.

doi:10.1074/jbc.M109.083493

26. Papachristou NI, Blair HC, Kalyvioti ES, Syggelos SA, Karavia EA, Kontogeorgakos V, et al. Western-type diet differentially modulates osteoblast, osteoclast, and lipoblast differentiation and activation in a background of APOE deficiency. Lab Invest. 2018;98(12):1516-1526. doi:10.1038/s41374-018-0107-7

27. Long MA, Rossi FM. Silencing inhibits Cre-mediated recombination of the Z/AP and Z/EG reporters in adult cells. PLoS One. 2009;4(5):e5435. doi:10.1371/journal.pone.0005435

28. Bao J, Ma HY, Schuster A, Lin YM, Yan W. Incomplete cre-mediated excision leads to phenotypic differences between Stra8-iCre; Mov10l1(lox/lox) and Stra8-iCre; Mov10l1(lox/ $\Delta$ ) mice. Genesis.

2013;51(7):481-490. doi:10.1002/dvg.22389

29. Vannella KM, Barron L, Borthwick LA, Kindrachuk KN, Narasimhan PB, Hart KM, et al. Incomplete deletion of IL-4Ra by LysM(Cre) reveals distinct subsets of M2 macrophages controlling inflammation and fibrosis in chronic schistosomiasis. PLoS Pathog. 2014;10(9):e1004372. doi:10.1371/journal.ppat.1004372

30. Bruderer M, Richards RG, Alini M, Stoddart MJ. Role and regulation of RUNX2 in osteogenesis. Eur Cell Mater. 2014;28:269-286. Published 2014 Oct 23. doi:10.22203/ecm.v028a19

380 31. Hwang SY, Foley J, Numaga-Tomita T, Petranka JG, Bird GS, Putney JW Jr. Deletion of Orai1 alters expression of multiple genes during osteoclast and osteoblast maturation. Cell Calcium. 2012;52(6):488-500. doi:10.1016/j.ceca.2012.10.001

32. Blair HC, Larrouture QC, Li Y, Lin H, Beer-Stoltz D, Liu L, et al. Osteoblast Differentiation and Bone Matrix Formation In Vivo and In Vitro. Tissue Eng Part B Rev. 2017;23(3):268-280. doi:10.1089/ten.TEB.2016.0454

33. Cheng Z, Li A, Tu CL, Maria CS, Szeto N, Herberger A, et al. Calcium-Sensing Receptors in Chondrocytes and Osteoblasts Are Required for Callus Maturation and Fracture Healing in Mice. J Bone Miner Res. 2020;35(1):143-154. doi:10.1002/jbmr.3864

34. Xi G, D'Costa S, Wai C, Xia SK, Cox ZC, Clemmons DR. IGFBP-2 stimulates calcium/calmodulindependent protein kinase kinase 2 activation leading to AMP-activated protein kinase induction which is required for osteoblast differentiation. J Cell Physiol. 2019;234(12):23232-23242. doi:10.1002/jcp.28890

35. Doroudi M, Plaisance MC, Boyan BD, Schwartz Z. Membrane actions of 1a,25(OH)2D3 are mediated by $\mathrm{Ca}(2+) / c a l m o d u l i n-d e p e n d e n t$ protein kinase II in bone and cartilage cells. J Steroid Biochem Mol Biol. 2015;145:65-74. doi:10.1016/j.jsbmb.2014.09.019 

A von Kossa for Bone Mineral

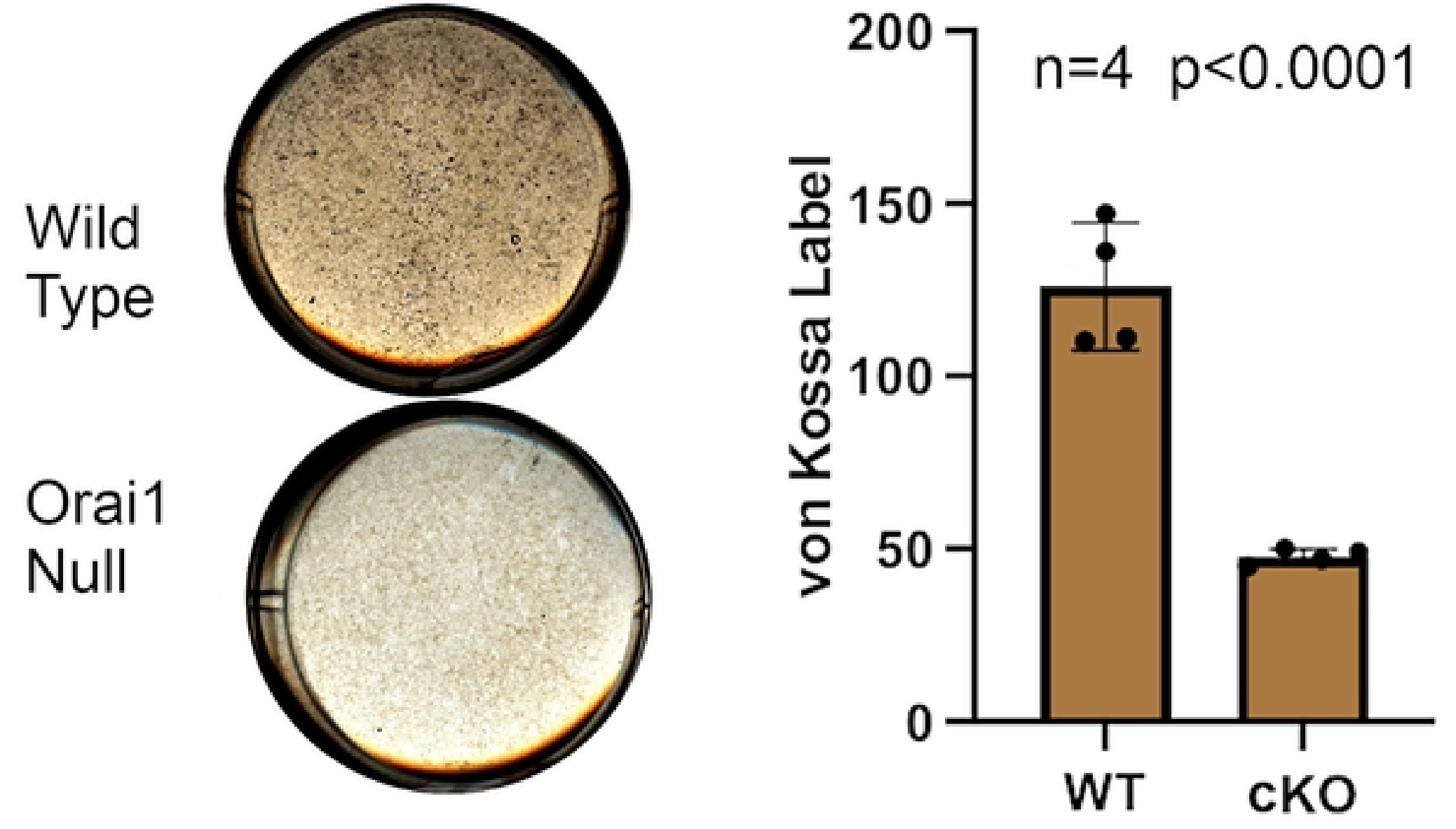

B Alkaline Phosphatase Activity

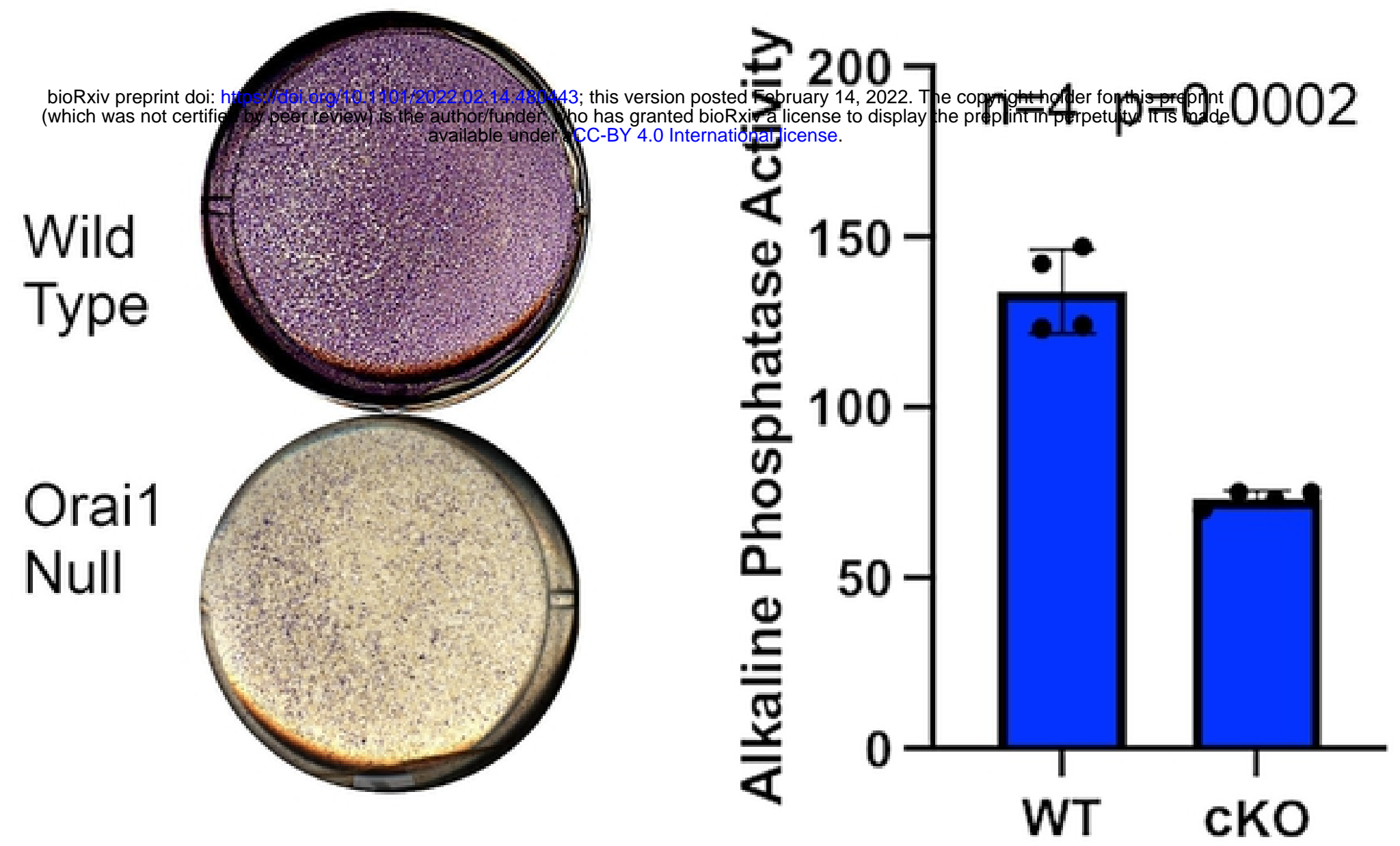

C Oil Red-O Stain for Adipocytes
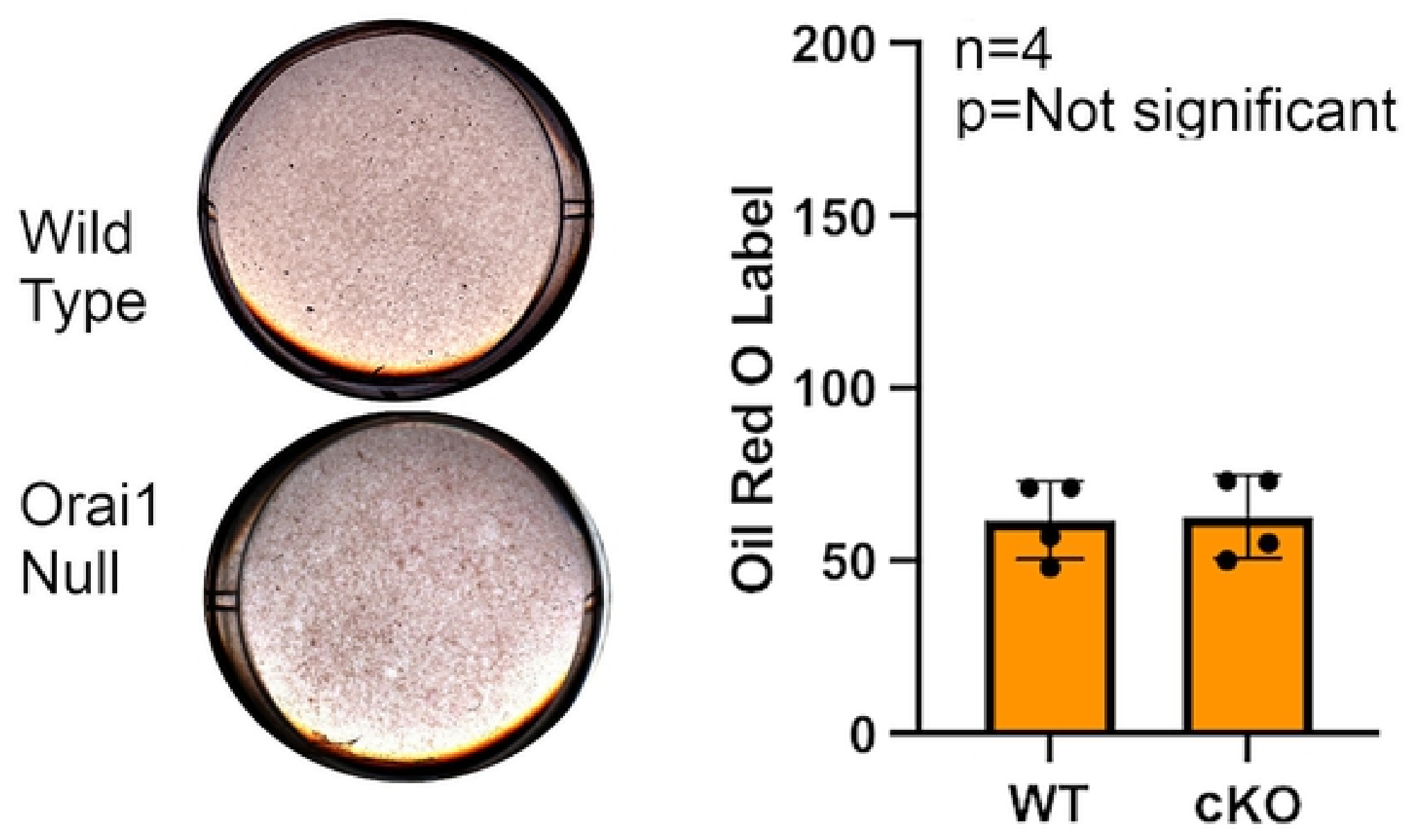

Figure 6 